\title{
No effect of $n-3$ long-chain polyunsaturated fatty acid (EPA and DHA) supplementation on depressed mood and cognitive function: a randomised controlled trial
}

\author{
Peter J. Rogers ${ }^{1}$, Katherine M. Appleton ${ }^{1}$, David Kessler ${ }^{2}$, Tim J. Peters ${ }^{2}$, David Gunnell ${ }^{3}$, \\ Robert C. Hayward ${ }^{1}$, Susan V. Heatherley ${ }^{1}$, Leonie M. Christian ${ }^{1}$, Sarah A. McNaughton ${ }^{4}$ and \\ Andy R. Ness ${ }^{5}$ \\ ${ }^{1}$ Department of Experimental Psychology, University of Bristol, UK \\ ${ }^{2}$ Academic Unit of Primary Health Care, Department of Community Based Medicine, University of Bristol, UK \\ ${ }^{3}$ Department of Social Medicine, University of Bristol, UK \\ ${ }^{4}$ MRC Human Nutrition Research, Cambridge, UK \\ ${ }^{5}$ Department of Oral and Dental Science, University of Bristol, UK \\ (Received 8 January 2007 - Revised 11 June 2007 - Accepted 20 June 2007)
}

\begin{abstract}
Low dietary intakes of the $n-3$ long-chain PUFA (LCPUFA) EPA and DHA are thought to be associated with increased risk for a variety of adverse outcomes, including some psychiatric disorders. Evidence from observational and intervention studies for a role of $n$-3 LCPUFA in depression is mixed, with some support for a benefit of EPA and/or DHA in major depressive illness. The present study was a double-blind randomised controlled trial that evaluated the effects of EPA + DHA supplementation $(1.5 \mathrm{~g} / \mathrm{d})$ on mood and cognitive function in mild to moderately depressed individuals. Of 218 participants who entered the trial, 190 completed the planned 12 weeks intervention. Compliance, confirmed by plasma fatty acid concentrations, was good, but there was no evidence of a difference between supplemented and placebo groups in the primary outcome namely, the depression subscale of the Depression Anxiety and Stress Scales at 12 weeks. Mean depression score was 8.4 for the $\mathrm{EPA}+$ DHA group and 9.6 for the placebo group, with an adjusted difference of -1.0 (95\% CI $-2 \cdot 8,0 \cdot 8 ; P=0.27)$. Other measures of mood, mental health and cognitive function, including Beck Depression Inventory score and attentional bias toward threat words, were similarly little affected by the intervention. In conclusion, substantially increasing EPA + DHA intake for 3 months was found not to have beneficial or harmful effects on mood in mild to moderate depression. Adding the present result to a meta-analysis of previous relevant randomised controlled trial results confirmed an overall negligible benefit of $n$-3 LCPUFA supplementation for depressed mood.
\end{abstract}

Depressed mood: Cognitive function: EPA: DHA

It is thought that in many countries current population intakes of the $n-3$ long-chain PUFA (LCPUFA; also known as long chain omega-3 fatty acids) EPA and DHA are lower than optimal for a variety of health outcomes ${ }^{1,2}$. In modern Western diets the principal source of these fatty acids is fish, particularly oily fish, which are relatively unpopular foods. Coupled with this there has been a rise in recent decades in intakes of oils and foods rich in $n-6$ LCPUFAs ${ }^{1}$. A decrease in the ratio of $n-3$ LCPUFA: $n-6$ LCPUFA intake in the diet has, in turn, been implicated in the aetiology of psychiatric disorders, including depression ${ }^{3-6}$, which itself is a major and increasing contributor to the global disease burden ${ }^{7}$.

DHA is a component of cell membrane phospholipids and its concentration can affect membrane fluidity and activity of membrane receptors and ion channels ${ }^{1,4}$. EPA is a precursor of DHA and of a variety of eicosanoid immune-neuroendocrine regulatory compounds, including PG, thromboxanes, prostacyclins and leukotrienes ${ }^{1,4}$ Animal studies have found effects of dietary concentrations of $n-3$ LCPUFA on phospholipid fatty acid composition and monoaminergic neurotransmission ${ }^{8,9}$.

In relation to depression, clinical studies have revealed associations between blood measures of fatty acid status and severity of depression ${ }^{10}$ and differences in fatty acid status between people with depression and age-matched controls ${ }^{11-13}$, in the direction of a lower $n$-3 LCPUFA concentration and lower $n-3$ LCPUFA: $n-6$ LCPUFA ratio in depression. Another study found an inverse relationship between adipose tissue DHA concentration and mild depression in a non-clinical sample ${ }^{14}$. Associations have also been noted between low dietary intakes of $n-3$ LCPUFA or fish and prevalence of depression across time and between and within countries ${ }^{3,15-17}$. On the other

Abbreviations: BDI, Beck Depression Inventory; DASS, Depression, Anxiety and Stress Scales; LCPUFA, long-chain PUFA; PUFAQ, PolyUnsaturated Fatty Acids Questionnaire.

* Corresponding author: Professor Peter J. Rogers, fax +440117 9288588, email Peter.Rogers@bristol.ac.uk 
hand, we found only a weak non-linear association between depressed mood and $n-3$ LCPUFA intake from fish, which appeared to be confounded by age and level of social and economic deprivation ${ }^{18}$. Hakkarainen et al. ${ }^{19}$ similarly reported no association between intakes of $n$-3 LCPUFA or fish and depressed mood and also no association with major depressive episodes or suicide. Another study found elevated levels of $n-3$ LCPUFA in patients with depression ${ }^{20}$.

Evidence from intervention studies investigating the effects of increasing $n$-3 LCPUFA intakes on depression is also mixed. A recent review of this evidence identified eighteen relevant randomised controlled trials, of which twelve met the criteria for inclusion in meta-analysis ${ }^{21}$. The evidence overall amounted to limited benefit of $n-3$ LCPUFA for depressed mood, although a beneficial effect was found in trials that enrolled clinical populations diagnosed with depressive illness. These latter trials, however, tended to be small ( $n$ 20-77) and the doses of $n-3$ LCPUFA (up to $9.6 \mathrm{~g} / \mathrm{d}$ ) associated with the largest effects in adults were very much higher than could be realistically achieved through diet.

The purpose of the present trial was to examine the effects of raising intakes of EPA and DHA on depressed mood in mild to moderately depressed individuals from the general population who were not receiving conventional treatments for depression. The participants were individuals who displayed evidence of depressed mood, but whose symptoms did not meet criteria for major depression. Anxiety, stress and anger were included as secondary outcomes due to their co-morbidity with depression ${ }^{22,23}$. Similarly, performance on various cognitive tasks was assessed because depressed mood is associated with impaired cognitive function ${ }^{24}$ and, independent of effects on mood, there is interest in possible benefits of $n-3$ LCPUFA supplementation for cognition ${ }^{5,25,26}$. The amounts of $n-3$ LCPUFA $(\mathrm{EPA}+\mathrm{DHA})$ provided in the supplement were substantially in excess of current UK dietary targets ${ }^{2}$, but potentially achievable through increased intake of oily fish and certainly so through the use of widely available fish oil supplements.

In summary, while a majority of previous trials have focused on individuals with severe depression in clinical settings and have mostly administered high amounts of $n-3$ LCPUFA, the present trial was concerned with investigating possible benefits in mild to moderate depression of an intake of $n$-3 LCPUFA that many in the general population might attain by appropriate changes in diet, supplement use or both. The prevalence of mild to moderate depression is substantial $^{27}$ and many people with mild to moderate depressive symptoms present in primary care, where there is concern about rising levels of prescribing conventional antidepressant treatments. Interest in finding safe alternative treatments with proven efficacy is therefore high. Appropriately, the main route of recruitment for the trial was through primary care settings; however, above all, the trial was designed to be relevant to the general population rather than the treatment of clinical (i.e. major) depression. It was hypothesised that supplementation with EPA + DHA for 12 weeks would improve mood and cognitive function.

\section{Methods and materials}

\section{Trial design and participants}

This was a single centre, double-blind randomised controlled trial that evaluated EPA + DHA supplementation $v$. placebo in 218 mildly moderate depressed individuals, of whom 190 completed the planned 12 weeks of supplementation.

A flow diagram summarising progress through the trial is presented in Fig. 1. Participants were recruited into the trial following two screens completed 2-4 weeks apart (screens 1 and 2). Screening questionnaires assessed demographics, EPA + DHA intake and depressed mood. EPA + DHA intake was assessed using a short dietary questionnaire, the PolyUnsaturated Fatty Acids Questionnaire (PUFAQ), developed specifically for the trial. It enquired about the recent (previous 3 months) frequency of intake of various sources of dietary fat, including white fish and oily fish, and intake of dietary supplements including fish oils. Responses were coded such that a score of 1 represented an EPA + DHA intake equivalent to consuming one portion of oily fish per week (based on data from previous studies ${ }^{2}$ ). Depressed mood was assessed using the short form of the Depression, Anxiety and Stress Scales (DASS) ${ }^{28}$. Two screens were undertaken to exclude those with transient disturbance of mood. Participants were suitable for the trial if at screens 1 and 2 they were no more than moderate consumers of EPA and DHA (PUFAQ score of $\leq 1.5$ ); suffering from mild to severe depressed mood (DASS depression score 10-24; scores of 21-27 are classified as 'severe' and $\geq 28$ as 'extremely severe'), aged 18-70 years old, not pregnant or breast feeding, not severely ill, not taking antidepressant medication for depression, not known to suffer from medical or other conditions that contraindicated use of the active or placebo supplement (e.g. gastrointestinal conditions, food intolerances, food allergies, special diets), fluent English speakers (because both the questionnaire and cognitive function measures were critically dependent on a good understanding of English).

Most of the participants were recruited via general practice surgeries in Bristol (Table 1). Researcher-based recruitment took place from May to September 2003 in thirteen general practice surgeries. For a randomly selected 2 -week period during this time, screening questionnaires were handed out by researchers to all persons in the waiting room between 08.30 and 11.00 hours. Potential participants completed the screening questionnaires and their willingness to take part in the trial was noted. Questionnaire box-based recruitment, for which posters highlighting the trial and recruitment questionnaires were placed in waiting rooms in forty-seven general practice surgeries, took place from September 2003 to December 2004. If desired, completed questionnaires could be returned by freepost to the research unit rather than being placed in the collection box. Further participants were recruited at two events, Science Week in a Bristol city centre shopping mall (March 2004) and Freshers' Fair at the University of Bristol (September 2004), and through advertising in the local media (newspapers and radio, January 2004) and around the University of Bristol (January, April and October 2004). These various methods of recruitment were undertaken to maximise participation of individuals with low mood who had not been labelled as depressed in a clinical setting. This recruitment strategy also helped to improve the generalisability of the trial. 


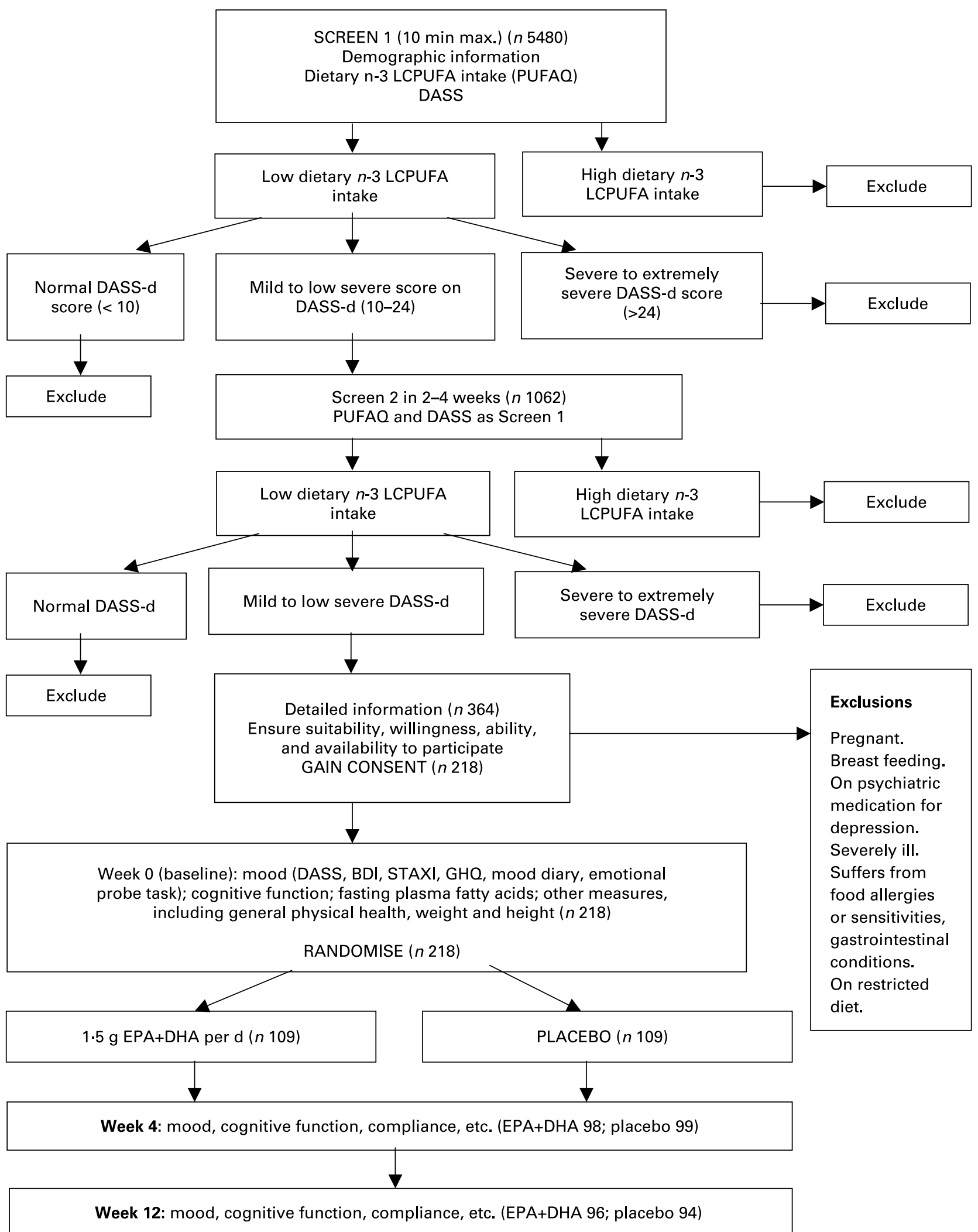

Fig. 1. Flow diagram of progress through the trial. LCPUFA, long-chain PUFA; PUFAQ, PolyUnsaturated Fatty Acids Questionnaire; DASS, Depression, Anxiety and Stress Scale; DASS-d, DASS depression; BDI, Beck Depression Inventory; STAXI, State-Traint Anger Expression Inventory; GHQ, General Health Questionnaire. For details of subjects and procedures, see Methods and materials. 
Table 1. Number of participants recruited into the trial using each method of recruitment†

\begin{tabular}{lcccc}
\hline & Researcher-based recruitment & Questionnaire box-based recruitment & Other methods & Total \\
\hline Number of people available & 5167 & Unknown & Unknown & Unknown \\
Number who completed questionnaires & 2982 & 2050 & 448 & 5480 \\
Number of volunteers & 1786 & 2050 & 448 & 4284 \\
Number of volunteers suitable (screen 1) & 387 & 557 & 118 & 1062 \\
Number of volunters suitable (screen 2) & 89 & 218 & 57 & 364 \\
Number recruited into the trial & 28 & 145 & 45 & 218 \\
\hline
\end{tabular}

* Via general practice surgeries.

†For details of subjects and procedures, see Methods and materials.

Informed consent was obtained from all participants prior to their inclusion in the trial. Approval for the trial was granted by Southmead Local Research Ethics Committee, United Bristol Healthcare Trust Research Ethics Committee, Frenchay Research Ethics Committee and NHS Primary Care Trusts for Bristol North and Bristol South and West.

\section{Intervention}

Participants were instructed to consume three capsules daily. These three capsules contained a total of either $630 \mathrm{mg}$ EPA, $850 \mathrm{mg}$ DHA, $870 \mathrm{mg}$ olive oil, $7.5 \mathrm{mg}$ mixed tocopherols and $12 \mathrm{mg}$ orange oil (EPA + DHA, the active treatment) or $2360 \mathrm{mg}$ olive oil, $7.5 \mathrm{mg}$ mixed tocopherols and $12 \mathrm{mg}$ orange oil (placebo treatment). The capsule casing was made from fish gelatine. Active and placebo capsules were identical in appearance. The orange oil was included to improve palatability and blinding. If bitten into, their predominant flavour was orange with an oily aftertaste. These capsules were purchased from Minami Nutrition SA (Antwerp, Belgium), who prepared them especially for the trial. They were supplied to participants in sealed, child-safe, plastic containers labelled with the participant's identification number.

\section{Outcome measures}

The primary outcome was depressed mood at 12 weeks follow-up, measured by the depression subscale of the DASS (long form) ${ }^{28}$. Secondary outcome measures were the Beck Depression Inventory (BDI) ${ }^{29}$, which is commonly used in clinical trials of treatments for depression, the anxiety and stress subscales of the DASS, the General Health Questionnaire $^{30}$, the state anger subscale of the State-Trait Anger Expression Inventory ${ }^{31}$, mood assessed using a diary and a visual probe task, and cognitive function assessed using various tasks. The DASS is suitable for the assessment of depression in general population samples and correlates fairly highly with the BDI ( $r 0.74)$, the difference in performance between these instruments being due largely to the inclusion of items in the BDI that assess somatic symptoms that do not appear to be strongly or uniquely related to depression $^{28}$.

For the mood diary, participants recorded their mood on ten clusters of descriptors (e.g. happy/cheerful/light-hearted, drowsy/sluggish/fatigued, clear-headed) rated on $100 \mathrm{~mm}$ unipolar line scales anchored at either end with 'not at all' and 'extremely'. Ratings were made at six pre-specified times $(08.00,11.00,13.00,16.00,18.00$ and 21.00 hours) daily for
3 d. A similar method was previously used successfully in a study of the effects of cholesterol-lowering on $\operatorname{mood}^{32}$. The visual probe task was based on methods described by MacLeod $\&$ Mathews $^{33}$ and Mogg \& Bradley ${ }^{34}$. It formed part of the battery of computer-presented cognitive tasks detailed later. Each trial of the visual probe task began with the appearance of a central fixation cross, which was replaced by two words, one appearing on the left and one on the right of the computer screen for $500 \mathrm{~ms}$. Next, immediately after the words disappeared, a left or right pointing arrow (the probe) appeared on the left or right of the screen and the participant had to indicate as quickly as possible with a key press the direction of the arrow. One of the words presented was physically or socially threatening in nature (e.g. coffin, pathetic) and one was neutral, matched for initial letter, word length, number of syllables and, as far as possible, frequency of general use, with the threatening word (e.g. collar, portable). The advantage of implicit (reaction time) measures of this type is that they are free from potential biases, such as impression management and social desirability, which can undermine the validity of mood questionnaires and interview methods.

Other aspects of cognitive function, including speed of information processing, reasoning using lexical and numerical cues, impulsivity and working memory were assessed using the following tasks: simple reaction time ${ }^{35}$; lexical decision ${ }^{36}$; digit-symbol substitution ${ }^{37}$; impulsivity ${ }^{35}$; N-Back ${ }^{38}$. Previous studies have found differences in performance between depressed and non-depressed individuals on all these tasks ${ }^{24,34,36,39,40}$ and some have reported improvement in performance alongside improvement in depressed mood during treatment for depression ${ }^{36,40}$. All cognitive tasks were programmed and presented using E-Prime 1.0 (Psychology Software Tools Inc., Pittsburgh, PA, USA) run on networked PC with 15-inch colour monitors and standard keyboards. They were undertaken in the afore-mentioned order, with the visual probe task occurring second to last, as a single battery lasting $45 \mathrm{~min}$ and were preceded on every occasion by a practice battery lasting 5-10 min, which contained all the tasks in shortened form. The purpose of this practice was to familiarise and re-familiarise participants with the tasks.

Compliance with the trial protocol was assessed using self-reported number of days on which capsules were taken, a count of the number of capsules returned and plasma fatty acid composition measured from fasting venous blood samples collected from all participants willing to provide blood. Analysis of fatty acid composition was carried out using GC. Total lipid was extracted from plasma with 2:1 (vol./vol.) 
chloroform and methanol. Fatty acid methyl esters were prepared by incubation of lipid with methanol containing $2 \%$ (v/v) sulphuric acid at $50^{\circ} \mathrm{C}$ for $2 \mathrm{~h}$. Fatty acid methyl esters were separated using a Hewlett Packard 6890 gas chromatograph (Agilent Technologies UK Ltd, Wokingham, Berks., UK) equipped with a $30 \mathrm{~m} \times 0.25 \mu \mathrm{m} \times 0.25 \mathrm{~mm}$ BPX70 fused silica capillary column (SGE, Milton Keynes, UK) and flame ionisation detection. The concentrations of individual fatty acids were determined by measurement of the peak area using ChemStation software (Agilent) and each fatty acid was expressed as a proportion of the total (see Burdge et $a .^{41}$ for further details).

Measurements were taken at baseline (week 0) and weeks 4 and 12 of supplementation. At each time point, participants were required to attend the Psychology Research Unit, University of Bristol starting at either 08.00 or 10.00 hours for $2 \mathrm{~h}$. A few days before, participants were sent the questionnaires, which were completed at home. These were returned and checked during their visit. On arrival, participants were first required to provide a blood sample and were weighed and their height measured. Participants were then given $30 \mathrm{~min}$ to consume breakfast. On their first visit, participants were free to consume whatever they chose for breakfast, but were requested to eat their usual breakfast if possible, and were asked to record this. On weeks 4 and 12, participants were asked to have this same breakfast to control for possible effects of breakfast on cognitive performance. Following the test batteries, participants were provided with their capsules and instructions on how to take them. Finally, participants were provided with a mood diary, to be completed over the following $3 \mathrm{~d}$ and returned by post. Participants were free to ask questions throughout the visit and were encouraged to telephone the researchers from home if necessary. Participants were contacted by telephone prior to each visit to confirm their appointment.

\section{Sample size}

A difference in means of between 3 and 4 points on the DASS depression scale (maximum range 42) was considered therapeutically meaningful, as such a decrease would be likely to result in the level of depression falling by one category (e.g. moderate to mild $)^{28}$. Using a standard deviation in DASS depression score of 7 points, for a power of $85 \%$ and a two-tailed significance level of 5\%, 200 participants would be needed to detect a difference in means of 3 DASS depression score points. For a difference of 4 points, power would be $98 \%$. To allow for attrition of $15-20 \%$, it was initially intended that 240 participants would be recruited.

\section{Randomisation and blinding}

Randomisation was stratified by gender, age $(<50$ and $\geq 50$ years) and DASS depression score at screen 2 (10-16 and 17-24), with computer-generated random allocations conducted using (randomly selected) blocks of six or eight. The supplier provided the capsules in sealed plastic containers (with the two groups distinguishable but blinded) and unique trial identification numbers were then attached by researchers not involved in participant recruitment or testing (T.J.P., D.G.) according to the allocation schedule. Randomisation of participants entering the trial was achieved through consecutive allocation of an identification number to each individual within each stratum by a trial researcher (K.M.A.). On provision of completed consent forms and baseline measures, the allocated container was unsealed and three capsules were removed to allow verification of treatment group at a later date if necessary.

The trial was presented to participants as a study of the effects of 'dietary supplements on health and well-being' and they were told that the capsules contained components of a natural healthy diet (e.g. vitamins and oils). Fish and fish oils were not mentioned explicitly in this context. Success of these blinding procedures was evaluated during debriefing by asking participants to identify whether they had received 'active' or 'placebo' supplements and then asking them what they thought the supplements had contained.

\section{Statistical methods}

Data analyses were performed using SPSS 12.0.1 (SPSS Inc., Chicago, IL, USA) and Stata Release 9 (StataCorp LP, College Station, TX, USA), commencing with descriptive statistics to describe and investigate comparability of the two randomised groups. The primary comparative analysis of the intervention was conducted on an intention-to-treat basis, employing regression to compare the outcomes between the groups at week 12 adjusting for baseline score for the outcome variable, age and gender. The method of last observation carried forward was used to replace the small number of missing values. Secondary analyses involved per-protocol regression models and interaction tests to compare the effects of supplementation across subgroups. These pre-specified subgroup analyses were based on DASS depression score at baseline $(<14 v . \geq 14$, which is the boundary between mild and moderate depression severity ${ }^{28}$ ).

The mood diary data were subjected to factor analysis (using the method of principal factoring with varimax rotation) to ascertain if there was a smaller number of underlying dimensions by which they could be characterised. Scores on the resulting latent variables were then subjected to the between-group comparisons described earlier using linear regression models (except that, due to larger amounts of missing data, baseline values were not included in these analyses).

\section{Results}

\section{Baseline characteristics and compliance}

Baseline characteristics of the participants were similar across the EPA + DHA and placebo groups (Table 2; Figs. 2, 3). Mean ages of the two groups were respectively 38.0 (SD 13.5) and 38.2 (SD 13.7) years and eighty-five $(78 \%)$ and eightythree $(76 \%)$ were female. The mean DASS depression score and age of the twenty-eight participants (thirteen in the $\mathrm{EPA}+\mathrm{DHA}$ group and fifteen in the placebo group) failing to complete their full course of supplements were $10 \cdot 2$ (SD 5.2) and 34.6 (SD 11.5) years, respectively, and twentyfour $(86 \%)$ were female. Reasons for non-completion of supplementation were: not having sufficient time to devote to the trial, given by six and seven participants (EPA + DHA and placebo respectively); the development of health problems 
Table 2. Depressed mood, cognitive function and other outcomes for EPA + DHA and placebo groups at pre-supplementation baseline and after 4 and 12 weeks (Values are means and standard deviations)

\begin{tabular}{|c|c|c|c|c|c|c|c|c|c|c|c|c|c|c|}
\hline & \multicolumn{4}{|c|}{ Baseline } & \multicolumn{4}{|c|}{ Week 4} & \multicolumn{4}{|c|}{ Week 12} & \multirow{3}{*}{$\begin{array}{c}\text { Difference between } \\
\text { means at week } 12^{*} \\
(95 \% \mathrm{Cl})\end{array}$} & \multirow{3}{*}{$\begin{array}{c}P \\
\text { value }\end{array}$} \\
\hline & \multicolumn{2}{|c|}{$E P A+D H A$} & \multicolumn{2}{|c|}{ Placebo } & \multicolumn{2}{|c|}{$E P A+D H A$} & \multicolumn{2}{|c|}{ Placebo } & \multicolumn{2}{|c|}{$E P A+D H A$} & \multicolumn{2}{|c|}{ Placebo } & & \\
\hline & Mean & SD & Mean & SD & Mean & SD & Mean & SD & Mean & SD & Mean & SD & & \\
\hline \multicolumn{15}{|l|}{ Intention-to-treat analysis ( $n$ 218) } \\
\hline DASS depression & 10.9 & $6 \cdot 1$ & $11 \cdot 0$ & 5.5 & 8.5 & $7 \cdot 3$ & 9.8 & $6 \cdot 5$ & 8.4 & $8 \cdot 0$ & $9 \cdot 6$ & $7 \cdot 3$ & $-1.02(-2.83,0.81)$ & 0.27 \\
\hline Beck Depression Inventory & 13.9 & $6 \cdot 3$ & 13.9 & $7 \cdot 2$ & $11 \cdot 1$ & 6.9 & $11 \cdot 2$ & $6 \cdot 6$ & $10 \cdot 6$ & 7.6 & 9.9 & 6.5 & $0.63(-0.98,2.24)$ & 0.44 \\
\hline $\begin{array}{l}\text { General Health } \\
\text { Questionnaire }\end{array}$ & 65 & 23 & 64 & 23 & 55 & 24 & 57 & 23 & 55 & 27 & 54 & 26 & $0.5(-5.7,6 \cdot 6)$ & 0.88 \\
\hline STAXI state anger & $12 \cdot 0$ & 3.6 & $12 \cdot 1$ & 3.9 & 11.9 & 3.4 & 11.8 & 3.5 & 11.6 & 3.4 & $12 \cdot 1$ & 5.3 & $-0.41(-1.49,0.67)$ & 0.46 \\
\hline DASS anxiety & $6 \cdot 0$ & $5 \cdot 7$ & $6 \cdot 0$ & $5 \cdot 1$ & 4.8 & $4 \cdot 3$ & $5 \cdot 2$ & 4.9 & $4 \cdot 3$ & 4.8 & $5 \cdot 1$ & 5.4 & $-0.73(-1.72,0.25)$ & 0.14 \\
\hline $\begin{array}{l}\text { DASS stress } \\
\text { Per protocol analysis (n 188) }\end{array}$ & $14 \cdot 9$ & $7 \cdot 8$ & $13 \cdot 6$ & 6.9 & $12 \cdot 9$ & $7 \cdot 4$ & $12 \cdot 7$ & $6 \cdot 4$ & 11.6 & $7 \cdot 6$ & 11.6 & $7 \cdot 2$ & $-0.81(-2.36,0.75)$ & 0.31 \\
\hline \multicolumn{2}{|c|}{ 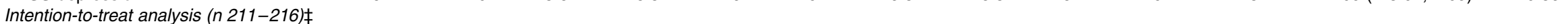 } & $6 \cdot 1$ & $11 \cdot 0$ & $5 \cdot 8$ & 8.5 & $7 \cdot 6$ & $9 \cdot 7$ & 6.5 & 8.5 & 8.4 & 9.4 & 7.5 & $-1.09(-3.02,1.09)$ & 0.36 \\
\hline $\begin{array}{l}\text { Visual probe bias } \\
\text { score }(\mathrm{ms})\end{array}$ & -3.9 & $23 \cdot 9$ & $-5 \cdot 3$ & $28 \cdot 5$ & 1.5 & $25 \cdot 7$ & $-1 \cdot 8$ & $28 \cdot 3$ & -0.5 & $25 \cdot 6$ & $-2 \cdot 3$ & $28 \cdot 8$ & $1.5(-5 \cdot 7,8 \cdot 8)$ & 0.68 \\
\hline Simple reaction time $(\mathrm{ms})$ & 370 & 75 & 381 & 56 & 386 & 95 & 397 & 90 & 391 & 85 & 398 & 71 & $3(-10,16)$ & 0.64 \\
\hline $\begin{array}{l}\text { Lexical decision (reaction } \\
\text { time; ms) }\end{array}$ & 763 & 181 & 774 & 174 & 736 & 177 & 725 & 145 & 718 & 160 & 717 & 133 & $9(-18,35)$ & 0.52 \\
\hline $\begin{array}{l}\text { Digit-symbol (reaction } \\
\text { time; } \mathrm{ms} \text { ) }\end{array}$ & 1648 & 401 & 1774 & 463 & 1563 & 378 & 1643 & 386 & 1537 & 391 & 1608 & 418 & $19(-36,75)$ & 0.50 \\
\hline Impulsivity, number of errors & $2 \cdot 81$ & $2 \cdot 40$ & 2.71 & 1.85 & 2.39 & 1.85 & 2.62 & 1.99 & $2 \cdot 36$ & 2.13 & 2.85 & 2.72 & $-0.55(-1.14,0.05)$ & 0.07 \\
\hline $\mathrm{N}$-back, number correct & 13.1 & $5 \cdot 9$ & 11.9 & 5.4 & 12.5 & 6.5 & 11.6 & $6 \cdot 3$ & $12 \cdot 1$ & 6.9 & 11.0 & 6.5 & $0.4(-1.1,1.9)$ & 0.58 \\
\hline
\end{tabular}

DASS, Depression, Anxiety and Stress Scales; STAXI, State-Trait Anger Expression Inventory

"Mean difference between treatment groups (i.e. EPA + DHA minus placebo) at week 12, adjusting for score at baseline and age and gender. Note that for all variables, lower scores signify better mood or cognitive function, excep for the N-back task where higher scores are better, and the visual probe task where zero scores indicate a lack of attentional bias.

tFor difference at week 12.

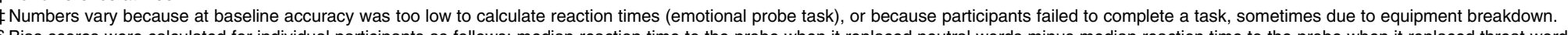

作

For details of subjects and procedures, see Methods and materials. 


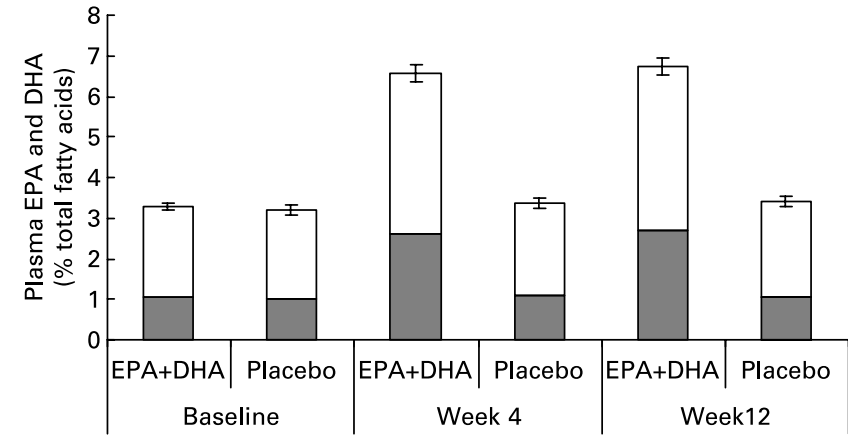

Fig. 2. Plasma EPA $(\square)$ and DHA ( $\square$ ) concentrations (\% total fatty acids) in EPA + DHA treatment and placebo groups at baseline and after 4 and 12 weeks of supplementation ( $n$ 185, 149 and 150, respectively). Data are means and error bars are standard errors of EPA + DHA \%. Between group differences in mean $(95 \% \mathrm{Cl})$ concentrations at week 12 , adjusting for values at baseline, age and gender, were: EPA, 1.53 (1.29, 1.77); DHA, 1.63 (1.41, 1.86); EPA + DHA, 3.16 (2.74, 3.58); all $P$ values $<0.00001$. For details of subjects and procedures, see Methods and materials.

unrelated to participation in the trial, given by two participants in each group; the development of health problems related to taking the capsules, given by two participants receiving placebo ('allergy' to citrus flavouring, intolerance of high fat content); one person became pregnant (placebo); one person left the country (EPA + DHA); four and three participants were not contactable following their previous visit.

Mean DASS depression at screen 2 of the 218 participants who subsequently entered the trial was 15.9 (SD 4.8) and their mean PUFAQ score was $0 \cdot 34$ (SD 0.52). At baseline, depressed mood had improved somewhat (Table 2); nevertheless, it remained above the euthymic ranges on both the DASS (scores of 10-13 are categorised as mild depression ${ }^{28}$ ) and BDI (scores of 10-18 are categorised as mild to moderate depression $\left.{ }^{29}\right)$. DASS depression at baseline was correlated

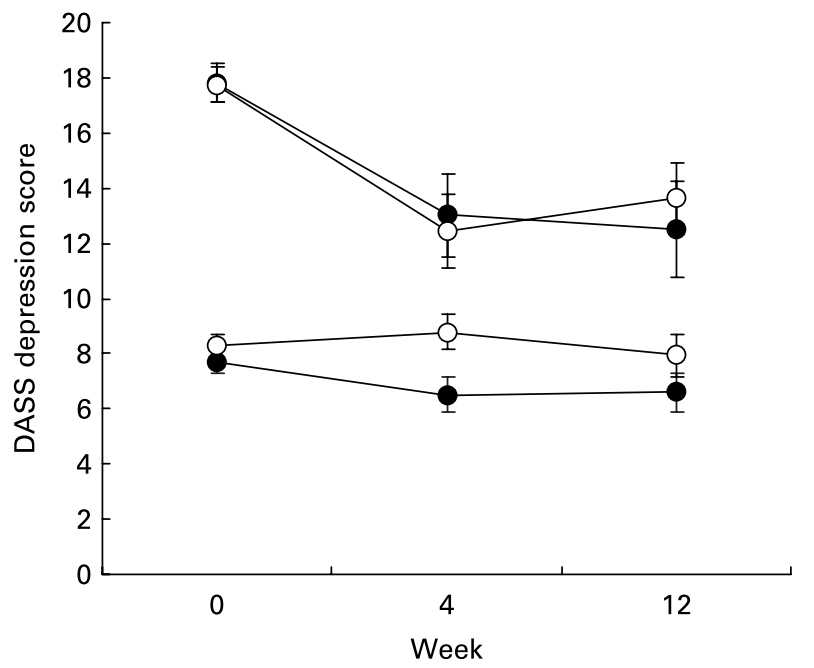

Fig. 3. Depression, Anxiety and Stress Scales (DASS) depression scores for $\mathrm{EPA}+\mathrm{DHA}(\bullet)$ and placebo groups $(O)$ as a function of week of treatment and level of depressed mood at baseline (week 0). Upper two lines, score at baseline $\geq 14$ ( $n$ 65); lower two lines, score at baseline $<14$ ( $n$ 153). For details of subjects and procedures, see Methods and materials. with BDI ( $r$ 0.62), General Health Questionnaire ( $r$ 0.66), State-Trait Anger Expression Inventory, state anger $(r$ 0.28), DASS anxiety $(r \quad 0.45)$ and DASS stress $\left(\begin{array}{ll}r & 0.55\end{array}\right)$ scores ( $n$ 218, all $P$ values $<0 \cdot 01$ ).

Results for compliance as measured by self-report (\% of days on which the capsules were consumed) and pill count at week 12 were, respectively, 98 (SD3) \% and 101 (SD11) $\%$ in the EPA + DHA group, and 98 (SD3) \% and 102 (SD11) $\%$ in the placebo group. Results are above $100 \%$ because pill counts indicated that some participants exceeded the prescribed dose. There were similarly high levels of compliance at week 4. From baseline to week 12, plasma $\mathrm{EPA}+\mathrm{DHA}$ concentration doubled in the EPA + DHA supplemented group $(P<0.00001)$, but did not change in the placebo group $(P=0 \cdot 16)$ (Fig. 2).

There was no evidence of relationships between baseline EPA and DHA concentrations, either alone or together, and any of the mental health measures; in particular, plasma $\mathrm{EPA}+\mathrm{DHA}$ concentration at baseline was not associated with baseline DASS depression ( $n$ 185, linear regression coefficient $-0.11(95 \% \mathrm{CI}-0.97,0.75 ; P=0.80))$.

\section{Mood - primary and secondary outcomes}

Mood and other aspects of mental health were not affected by EPA + DHA supplementation (Table 2). Specifically, the primary outcome of depressed mood improved over the 12 weeks of the intervention, but to a similar extent in EPA + DHA and placebo groups. All the values in the $95 \%$ CI for the adjusted difference in DASS depression score are smaller than those specified as therapeutically significant in the a priori sample size calculation.

Analyses based on data only from participants who fully completed the trial also showed no effects of the intervention, as did per protocol analyses that additionally omitted two EPA + DHA participants who had high plasma $\mathrm{EPA}+\mathrm{DHA}$ concentrations at baseline due to fish oil supplement use (which was not recognised until debriefing at the end of the trial). Per protocol results for DASS depression are shown in Table 2.

Regarding the subgroup analysis, while DASS depression scores fell to a greater extent in the subgroup with higher scores at baseline (Fig. 3), there was no evidence of a differential effect of EPH + DHA across the two subgroups (week 12 supplementation by depression group interaction, $P=0 \cdot 90$ ). Results were similar for the BDI, with participants in the higher subgroup scoring on average at the top of the mild to moderate depression BDI cut-off at baseline and remaining within this range at week 12 , but with no differential effect of supplementation $(P=0.65)$.

The factor analysis performed on the mood diary data identified three components with eigenvalues $>1$, which corresponded to 'dysphoric mood' (relaxed/calm (-), bad mood $(+)$, happy/cheerful/light-hearted $(-)$, tense/anxious/nervous/on edge/stressed $(+)$, sad/gloomy/miserable $(+))$, 'drowsiness' and 'alertness and clear-headedness'. Not all participants successfully completed their mood diaries. Analysable data were available for seventy-seven EPA + DHA and seventy-four placebo participants at week 12. Drowsiness and alertness and clear-headedness, but not dysphoric mood, varied as a function of time of day $(P<0.001)$. All three 
mood components were found to be unaffected by supplementation ( $P$ values $>0.1$ for effects of supplementation and supplementation by time of day interaction effects). Results for dysphoric mood were as follows: EPA + DHA 34.7 (SD 13.4); placebo 35.0 (SD 11.9); adjusted difference between means $-0.2(95 \% \mathrm{CI}-4 \cdot 2,3 \cdot 8 ; P=0.93)$.

\section{Cognitive function}

There was no evidence of effects of supplementation on cognitive function (Table 2). Although there was a suggestion that performance on the impulsivity task might be improved by $\mathrm{EPA}+\mathrm{DHA}$, the number of tests performed means that this should be regarded with caution.

The negative emotional probe bias scores at baseline indicate a propensity to avoid attending to threat words $(P=0.012$ from a $t$ test for all 211 individuals combined). This propensity was stronger in more depressed participants (bias scores -11.9 (SD 22.2) and -1.5 (SD 27.3) $\mathrm{ms}$ for DASS depression at baseline $\geq 14$ and $<14$, respectively; difference between means adjusted for age and gender -10.4 (95\% CI $-2.7,-18 \cdot 1 ; P=0.009)$; however, supplementation did not affect this measure in either subgroup (all $P$ levels $>0 \cdot 1$ ).

\section{Concealment of the intervention}

Participants were no better than chance at identifying which treatment they had received and this did not differ between groups (numbers saying 'active' and 'placebo' respectively, were thirty-eight and fifty-eight for the EPA + DHA group and thirty-one and sixty-three for the placebo group; $P=0.34$ from a $\chi^{2}$ test). Twelve participants said that they had received cod liver oil, fish oil or $n-3$ oils, of which ten were in the EPA + DHA group. Other guesses at what the capsules contained included vitamins (thirty-nine), minerals (seven), oils (five) and herbal extracts (three).

\section{Discussion}

The main finding of the present trial is the lack of a therapeutically important effect of $n$-3 LCPUFA supplementation on depressed mood among people with mild to moderate depression. The confidence interval for the primary outcome (DASS depression score) excluded both a therapeutically beneficial effect and a harmful effect. Similarly, other measures of depressed mood were not improved by EPA + DHA over placebo. Indeed, all measured aspects of mood and mental health, including anxiety, stress and cognitive function, were unaffected by supplementation, even in the subgroup with a higher level of depression on entry into the trial. For this subgroup, which at baseline scored in the range of moderate to severe depression on the DASS, there was not even a slight trend toward a benefit of EPA + DHA supplementation.

To the authors' knowledge, this is the largest trial investigating the effects of $n-3$ LCPUFA supplementation on depressed mood to be completed to date. Great care was taken to conceal the specific nature of the intervention and blinding was successful. The EPA + DHA supplement was well tolerated and the loss to follow-up of $13 \%$ was lower than anticipated for a non-clinical group participating in a lengthy protocol. It is unlikely that the absence of beneficial effects can be explained by the level or duration of supplementation. Compliance appeared to be good and after 12 weeks plasma EPA + DHA concentration in the supplemented group was twice that of the placebo group. A similar effect on blood concentrations of EPA + DHA was reported recently in a trial using a higher level of EPA + DHA supplementation for 12 weeks ${ }^{42}$. Even with much poorer compliance, supplementation would have increased $n$-3 LCPUFA intake to above levels consistent with UK dietary recommendations. Recent UK recommendations are for adults to consume at least two portions of fish per week, one of which should be oily (equivalent to about 3 g EPA + DHA per week) $)^{2}$. Current intakes, especially of oily fish, are considerably lower than this ${ }^{2}$.

The duration of supplementation in this trial was in line with previous work in mental health. Three studies of $n-3$ LCPUFA found benefits for depressed mood of supplementation for 12 weeks or less ${ }^{43-45}$. Furthermore, in people with bipolar disorder, changes in $\mathrm{T}_{2}$ whole brain relaxation times, indicative of changes in neuronal membrane fluidity, have been found after 4 weeks EPA + DHA supplementation ${ }^{46}$. Evidence from this study, however, was somewhat less clear for the lowest dose investigated $(2 \mathrm{~g})$ and there did not appear to be an effect on depression.

At baseline in the present trial, there was attentional avoidance of the threat words (e.g. injury, violence, failure, criticised) presented in the visual probe task. This avoidance was more marked in the more depressed group. While attentional vigilance for threat-related stimuli is commonly found among those with generalised anxiety disorder, results for depression are less clear, with relatively few previous reports of visual probe tasks revealing attentional biases in depression ${ }^{34}$. Similar to the results for the self-report measures of depressed mood (DASS depression and BDI), attentional avoidance of threat words declined over the 12 weeks of the trial and again there was no effect of supplementation, even for the more depressed group. In view of the interest in possible effects of $n$-3 LCPUFA in the treatment of impulsivity disorders' ${ }^{6}$, it is noteworthy that EPA + DHA $v$. placebo brought about a marginal improvement in performance on the impulsivity task. However, this result must be treated with considerable caution, as the number of secondary outcomes examined in the analyses means there is a correspondingly high probability of a Type I error (that is, observing a difference by chance). Furthermore, even if it is confirmed, the extent to which such an effect might predict a benefit for impulsive behaviour generally or in relation to Attention Deficit Hyperactivity Disorder, aggression or even suicide risk $^{4}$ is far from certain.

The evidence from this trial therefore does not favour a benefit for depressed mood or cognitive function of increased $n-3$ LCPUFA intake. An association between dietary intake of $n-3$ LCPUFA and depressed mood has been found in various populations ${ }^{16,17}$; however, analysis of cross-sectional data on nearly 3000 of the people screened during recruitment for the present trial did not confirm such a relationship when level of social and economic deprivation and age were taken into account ${ }^{18}$. Furthermore, depressed mood among the participants entering the trial was not related to plasma $n-3$ LCPUFA concentrations. Adding the present trial to a meta-analysis of the other randomised controlled trials on this subject ${ }^{21}$ confirms an overall 
Trial

Stoll et al. 47

Fenton et al. 48

Nemets et al. 43

Peet \& Horrobin (dose $1 \mathrm{~g} / \mathrm{d})^{44}$

Peet \& Horrobin (dose $2 \mathrm{~g} / \mathrm{d}$ ) 44

Peet \& Horrobin (dose $4 \mathrm{~g} / \mathrm{d})^{44}$

Llorente et al. 49

Marangell et al.50

Ness et al. 51

Su et al. 45

Zanarini \& Frankenburg 52

Silvers et al. 42

Frangou et al. (dose $1 \mathrm{~g} / \mathrm{d})^{53}$

Frangou et al. (dose $2 \mathrm{~g} / \mathrm{d})^{53}$

Nemets et al.54

Present trial

negligible effect size for $n$-3 LCPUFA supplementation $v$. placebo (Fig. 4). This meta-analysis also reveals evidence of heterogeneity and substantial funnel plot asymmetry, probably due to publication bias and/or differences in trial populations ${ }^{21}$.

The participants for the present trial were recruited from community-based settings and in general had mild to moderate depressive symptoms. Those previous studies that have shown benefits had much smaller sample sizes and enrolled individuals with more severe depressive symptoms from clinical settings. It remains that the present randomised trial is the largest and most relevant to the general population. It did not provide any evidence to support the use of $n$-3 LCPUFA to improve depressed mood.

\section{Acknowledgements}

Supported by a grant (NO5038) from the Food Standards Agency, UK government and by the University of Bristol, UK. The authors thank the participants who gave their time to the trial, general practitioners and others who facilitated participant recruitment, Professor Philip Calder and Annette West (University of Southampton) for the plasma fatty acid analyses and Dr Caroline Bolton-Smith (MRC-HNR Cambridge) for initial planning and design of the dietary aspects of the trial. of subjects and procedures, see Appleton et al. ${ }^{21}$ and Methods and materials.

\section{Standardised mean \% weight} difference $(95 \% \mathrm{Cl})$

$\begin{array}{rr}1.39(0.58,2.20) & 1.9 \\ 0.09(-0.33,0.51) & 6.9 \\ 1.06(0.11,2.01) & 1.4 \\ 0.60(-0.08,1.28) & 2.6 \\ 0.06(-0.60,0.71) & 2.9 \\ 0.27(-0.39,0.94) & 2.8 \\ -0.15(-0.57,0.26) & 7.1 \\ 0.81(0.13,1.50) & 2.6 \\ -0.20(-0.39,-0.02) & 35.8 \\ 1.91(0.99,2.82) & 1.5 \\ 0.34(-0.42,1.11) & 2.1 \\ -0.23(-0.68,0.22) & 6.0 \\ 0.69(0.12,1.27) & 3.7 \\ 0.53(-0.03,1.09) & 3.9 \\ 1.74(0.85,2.63) & 1.5 \\ -0.10(-0.36,0.17) & 17.3\end{array}$

$0.09(-0.02,0.20)$

Fig. 4. Effect of $n-3$ long-chain PUFA (LCPUFA) on depressed mood. Forest plot showing individual and combined effect size estimates and $95 \%$ Cl for the twelve trials included in the meta-analysis (fixed effects model, standardised mean effect calculated using Hedges' adjusted $g$ ) conducted by Appleton et al. ${ }^{21}$

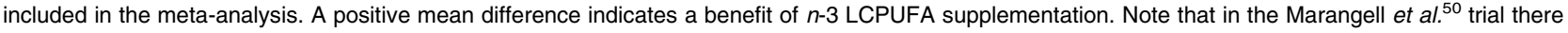
substantial differences in depression scores between placebo and treatment groups at baseline, which when adjusted for greatly reduces the treatment effects size, leading the authors to conclude that their trial 'failed to show a significant effect of DHA monotherapy in subjects with major depression'. For details

\section{References}

1. Simopoulos AP (1999) Essential fatty acids in health and chronic disease. Am J Clin Nutr 70, Suppl, 560S-569S.

2. SACN/COT (2004) Advice on Fish Consumption: Benefits and Risks. London: TSO.

3. Hibbeln JR \& Salem N (1995) Dietary polyunsaturated fatty acids and depression: when cholesterol does not satisfy. Am $J$ Clin Nutr 62, 1-9.

4. Peet M, Glen I \& Horrobin DF (1999) Phospholipid Spectrum Disorder in Psychiatry. Carnforth Lancashire: Marius Press.

5. Rogers PJ (2001) A healthy body, a healthy mind: long-term impact of diet on mood and cognitive function. Proc Nutr Soc 60, 135-143.

6. Hallahan B \& Garland MR (2004) Essential fatty acids and their role in the treatment of impulsivity disorders. Prost Leuk Ess Fatty Acids 71, 211-216.

7. Murray CJL \& Lopez AD (1996) The Global Burden of Disease. Cambridge MA: Harvard School of Public Health.

8. Chalon S, Delion-Vancassel S, Blezung C, Guilloteau D, Leguisquet AM, Besnard JC \& Durand G (1998) Dietary fish oil affects monoaminergic neurotransmission and behaviour in rats. J Nutr 128, 2512-2519.

9. Zimmer L, Delion-Vancassel S, Durand G, Guilloteau D, Bodard S, Besnard JC \& Chalon S (2000) Modification of dopamine neurotransmission in the nucleus accumbens of rats deficient in n-3 polyunsaturated fatty acids. J Lipid Res $41,32-40$. 
10. Adams PB, Lawson S, Sanigorski A \& Sinclair AJ (1996) Arachidonic to eicosapentaenoic acid ratio in blood correlates positively with clinical symptoms of depressed mood. Lipids 31, S167-S176.

11. Peet M, Murphy B, Shay J \& Horrobin D (1998) Depletion of omega-3 fatty acid levels in red blood cell membranes of depressive patients. Biol Psychiatry 43, 315-319.

12. Maes M, Christophe A, Delanghe J, Altamura C, Neels H \& Meltzer HY (1999) Lowered omega-3 polyunsaturated fatty acids in serum phospholipids and cholesteryl esters of depressed patients. Psychiat Res 85, 275-291.

13. Tiemeier H, van Tuijl HR, Hofman A, Kiliaan AJ \& Breteler MM (2003) Plasma fatty acid composition and depression are associated in the elderly: the Rotterdam Study. Am J Clin Nutr 78, 40-46.

14. Mamalakis G, Tornaritis M \& Kafatos A (2002) Depression and adipose essential polyunsaturated fatty acids. Prost Leuk Ess Fatty Acids 67, 311-318.

15. Noaghiul S \& Hibbeln JR (2003) Cross-national comparisons of seafood consumption and rates of bipolar disorders. Am J Psychiatry 160, 2222-2227.

16. Silvers KM \& Scott KM (2002) Fish consumption and selfreported physical and mental health status. Public Health Nutr 5, 427-431.

17. Tanskanen A, Hibbeln JR, Tuomilehto J, Uutela A, Haukkala A, Viinamaki H, Lehtonen $J$ \& Vartiainen E (2001) Fish consumption and depressive symptoms in the general population in Finland. Psychiatric Services 52, 529-531.

18. Appleton KM, Peters TJ, Hayward RC, Heatherley SV, McNaughton SA, Gunnell D, Rogers PJ, Ness AR \& Kessler D (2007) Depressed mood and $n 3$ polyunsaturated fatty acid intake from fish: Non-linear or confounded association? Soc Psychiatry Psychiatr Epidemiol 42, 100-104.

19. Hakkarainen R, Partonen T, Haukka J, Virtamo J, Albanes D \& Lonnqvist J (2004) Is low dietary intake of omega-3 fatty acids associated with depression? Am J Psychiatry 161, $567-569$.

20. Ellis FR \& Sanders TA (1977) Long-chain polyunsaturated fatty acids in endogenous depression. J Neurol Neurosur Psychiatry 40, $168-169$.

21. Appleton KM, Hayward RC, Gunnell D, Peters TJ, Rogers PJ, Kessler D \& Ness AR (2006) Effects of n-3 long-chain polyunsaturated fatty acids on depressed mood: systematic review of published trials. Am J Clin Nutr 84, 1308-1316.

22. Lovibond PF \& Lovibond SH (1995) The structure of negative emotional states: Comparison of the Depression Anxiety Stress Scales (DASS) with the Beck depression and anxiety inventories. Behav Res Therapy 33, 335-343.

23. Winkler D, Pjrek E \& Kasper S (2005) Anger attacks in depression: evidence for a male depressive syndrome. Psychother Psychosom 74, 303-307.

24. Austin MP, Mitchell P \& Goodwin GM (2001) Cognitive deficits in depression: possible implications for functional neuropathy. Brit J Psychiatry 178, 200-206.

25. Kalmijn S, van Boxtel MJP, Ocké M, Verschuren WMM, Kromhout D \& Launer LJ (2004) Dietary intake of fatty acids and fish in relation to cognitive performance at middle age. Neurology 62, 275-280.

26. Fontani G, Corradeschi F, Felici A, Alfatti F, Migliorini S \& Lodi L (2005) Cognitive and physiological effects of omega-3 polyunsaturated fatty acid supplementation in healthy subjects. Eur J Clin Invest 35, 691-699.

27. Jenkins R, Lewis G, Bebbington P, Brugha T, Farrell M, Gill B \& Meltzer H (1997) The National Psychiatric Morbidity Surveys of Great Britain: initial findings from the Household Survey. Psychol Med 27, 755-789.
28. Lovibond SH \& Lovibond PF (1995) Manual for the Depression Anxiety and Stress Scales. Sydney: Psychology Foundation of Australia Inc.

29. Beck AT \& Steer RA (1987) Beck Depression Inventory Manual. San Antonio, Texas: Psychological Corporation.

30. Goldberg D \& Williams P (1988) A User's Guide to the General Health Questionnaire. Basingstoke, Hampshire: NFER-Nelson Publishing.

31. Spielberger CD (1991) State-Trait Anger Expression Inventory: Professional Manual. Odessa, Florida: Psychological Assessment Resources Inc.

32. Polet P, Rogers PJ, Wardle J, Atkinson EA, Vallis L, Rapoport L, Taylor M \& Judd P (1998) Mood effects of reduced-fat dietary treatment. Proc Nutr Soc 57, 83A.

33. MacLeod C \& Mathews A (1988) Anxiety and the allocation of attention to threat. $Q J$ Exp Psychol 40, 653-670.

34. Mogg K \& Bradley BP (2005) Attentional bias in generalized anxiety disorder versus depressive disorder. Cognitve Ther Res 29, 29-45.

35. Rogers PJ, Heatherley SV, Hayward RC, Seers HE, Hill J \& Kane M (2005) Effects of caffeine and caffeine withdrawal on mood and cognitive performance degraded by sleep deprivation. Psychopharmacology 179, 742-752.

36. Brown RG, Scott LC, Bench CJ \& Dolan RJ (1994) Cognitive function in depression: its relationship to the presence and severity of intellectual decline. Psychol Med 24, 829-847.

37. Weschler D (1997) Weschler Adult Intelligence Scale, 3rd ed., San Antonio, TX: Psychological Corporation.

38. Baddeley A (1986) Working Memory. Oxford: Clarendon Press.

39. Rabbitt P, Donlan C, Watson P, McInnes L \& Bent L (1995) Unique and interactive effects of depression, age, socio-economic advantage and gender on cognitive performance of normal healthy older people. Psychol Aging 10, 307-313.

40. Sackeim HA, Keilp JG, Rush AJ, et al. (2001) The effects of vagus nerve stimulation on cognitive performance in patients with treatment-resistant depression. Neuropsychiatr Neuropsychol Behav Neurol 14, 53-62.

41. Burdge GC, Wright P, Jones AE \& Wootton SA (2000) A method for separation of phosphatidylcholine, triacylglycerol, non-esterified fatty acids and cholesterol esters from plasma by solid-phase extraction. Brit J Nutr 84, 781-787.

42. Silvers KM, Woolley CC, Hamilton FC, Watts PM \& Watson RA (2005) Randomized double-blind placebo-controlled trial of fish oil in the treatment of depression. Prost Leuk EsS Fatty Acids 72, 211-218.

43. Nemets B, Stahl Z \& Belmaker RH (2002) Addition of omega-3 fatty acid to maintenance medication treatment for recurrent unipolar depressive disorder. Am J Psychiatry 159, 477-479.

44. Peet MB \& Horrobin DF (2002) A dose-ranging study of the effects of ethyl-eicosapentaenoate in patients with ongoing depression despite apparently adequate treatment with standard drugs. Arch Gen Psychiatry 59, 913-919.

45. Su K-P, Huang S-Y, Chiu C-C \& Shen WW (2003) Omega-3 fatty acids in major depressive disorder - a preliminary double-blind placebo-controlled trial. Eur Neuropsychopharm 13, 267-271

46. Hirashima F, Parow AM, Stoll AL, Demopulos CM, Damico KE, Rohan ML, Eskesen JG, Zuo CS, Cohen BM \& Renshaw PF (2004) Omega-3 fatty acid treatment and T2 whole brain relaxation times in bipolar disorder. Am J Psychiatry 161, 1922-1924.

47. Stoll AL, Severus WE, Freeman MP, Rueter S, Zboyan HA, Diamond E, Cress KK \& Marangell LB (1999) Omega-3 fatty acids in bipolar disorder - a preliminary double-blind, placebo-controlled trial. Arch Gen Psychiatry 56, 407-412.

48. Fenton WS, Dickerson F, Boronow J, Hibbeln JR \& Knable M (2001) A placebo-controlled trial of omega-3 fatty acid 
(ethyl eicosapentaenoic acid) supplementation for residual symptoms and cognitive impairment in schizophrenia. Am J Psychiatry 158, 2071-2074.

49. Llorente AM, Jensen CL, Voigt RG, Fraley JK, Berretta MC \& Heird WC (2003) Effect of maternal docosahexaenoic acid supplementation on postpartum depression and information processing. Am J Obstet Gynecol 188, 1348-1353.

50. Marangell LB, Martinez JM, Zboyan HA, Kertz B, Seung Kim HF \& Puryear LJ (2003) A double-blind, placebo-controlled study of the omega-3 fatty acid docosahexaenoic acid in the treatment of major depression. Am J Psychiatry 160, 996-998.

51. Ness AR, Gallacher JEJ, Bennett PD, Gunnell DJ, Rogers PJ, Kessler D \& Burr ML (2003) Advice to eat fish and mood: a randomised controlled trial in men with angina. Nutr Neurosci 6, 63-65.

52. Zanarini MC \& Frankenburg MD (2003) Omega-3 fatty acid treatment of women with borderline personality disorder: a double-blind, placebo-controlled pilot study. Am J Psychiat 160, 167-169.

53. Frangou S, Lewis M \& McCrone P (2006) Efficacy of ethyleicosapentaenoic acid in bipolar depression: randomised double-blind placebo-controlled study. Brit J Psychiatry 188, $46-50$.

54. Nemets H, Nemets B, Apter A, Bracha Z \& Belmaker RH (2006) Omega-3 treatment of children's depression: a controlled, double-blind pilot study. Am J Psychiatry 163, $1098-1100$. 